\title{
Magnetron Injection Gun for high-power gyroklystron
}

\author{
Liang Zhang, Laurence J.R. Nix, and Adrian W. Cross
}

\begin{abstract}
A magnetron injection gun (MIG) can generate an annular electron beam with a high transverse-to-axial velocity ratio for gyrotron devices. This paper compares the different configurations of the MIGs and their suitable applications were analyzed from the theoretical study. Following that, a MIG for a $48 \mathrm{GHz}, 2 \mathrm{MW}$ output power gyroklystron was designed and optimized by parameterizing the MIG's geometry and the magnetic field. By using the standard triode-type configuration, a low alpha spread of $8.9 \%$ was achieved. The simulation results showed that the magnetic field profile also plays an important role in the MIG design. The angle of the magnetic field on the emitter surface affects the alpha value and the alpha spread, which was not able to be predicted by the synthesis method. It provides an extra degree of freedom for tuning the MIG's performance in the experiment where the geometry of the gun is fixed.
\end{abstract}

Index Terms - magnetron injection gun, gyroklystron, velocity spread, small orbit beam.

\section{INTRODUCTION}

Gyrotron devices based on cyclotron resonance maser (CRM) instability are capable to generate high power radiation at high frequency [1, 2]. The dominant beam-wave energy exchange is in the azimuthal direction with the TE mode in the interaction circuit. An annular electron beam with a fraction of the electron's momentum in the transverse direction while propagating in a uniform guiding magnetic field is required for effective interaction. Such a beam can be generated by a magnetron injection type of electron gun (MIG) [3], where the electrons travel through an adiabatic magnetic field compression region. The guiding center of the electrons from a MIG is normally off-center. A special case is the axis-encircling beam, which is also called a large-orbit beam, which can be generated by an electron gun with a cusp magnetic field [4, 5].

There are different configurations of MIGs [6-8], including the diode type, standard and inverse-cathode triode-type guns which have an additional modulation anode, as shown in Fig. 1. Although they are all able to generate a small orbit electron beam, they have their unique properties and are suitable for different applications. The diode MIG has the simplest geometry and a compact structure. It is easy to achieve a robust design and smaller assembly tolerance. The triode structure has an additional electrode with a potential difference from the

L. Zhang (liang.zhang@strath.ac.uk), L. J. R. Nix (laurence.nix@strath.ac. uk) and A. W. Cross (a.w.cross@strath.ac.uk) are with Department of Physics, SUPA, University of Strathclyde, Glasgow, G4 0NG, Scotland, UK and the Cockcroft Institute, Warrington Bank Quay. UK. This work was supported by the European Commission Horizon 2020 Project "CompactLight" under Grant 777431-XLS and in part by the Science and Technology Facilities Council cathode and the anode, which requires an extra power supply and electric insulation which makes it more complicated to construct and assemble. However, it provides an additional degree of freedom (the potential on the modulation anode) to further adjust the electron beam quality in operation. In an inverse-cathode MIG (IMIG), the modulation anode has a smaller radius than the emitter. Due to the constrain of electric break-down between the electrodes, the IMIG is suitable for gyrotron devices which have a larger emitter radius, therefore, allowing sufficient insulation gap distance. The IMIG is also a coaxial structure therefore more suitable for gyrotron devices that use a coaxial interaction circuit [9]. It has the advantage of a larger cathode radius (consequently large beam current), but is more challenging to align and assemble, therefore, it is not commonly used. The other two types of MIGs are more common and widely used in gyrotron devices with cylindrical interaction circuits.

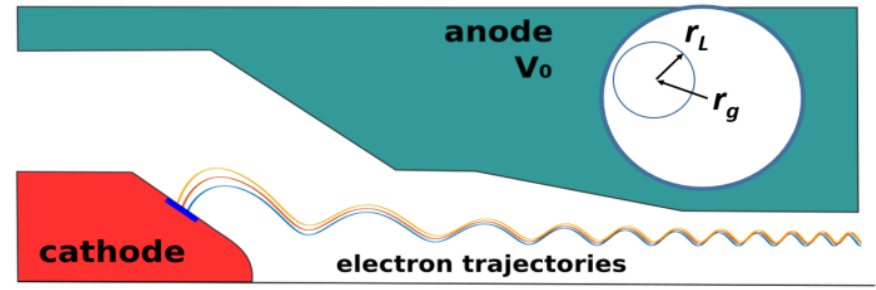

(a)

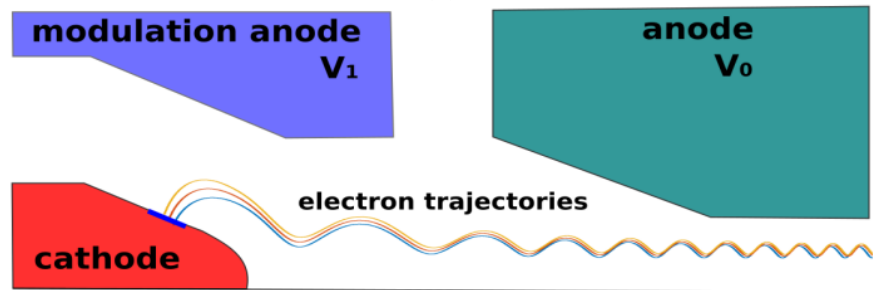

(b)

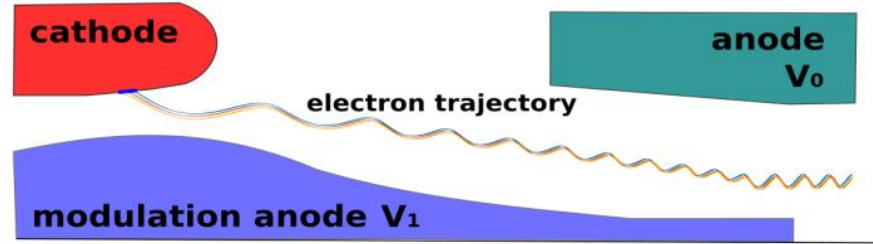

(c)

Fig. 1 different types of MIGs, (a) diode-type, (b) standard triode-type, (c) inverse-cathode MIG.

(STFC) U.K., Cockcroft Institute Core under Grant R160525-1, and in part by the U.K. Engineering and Physical Sciences Research Council (EPSRC) under Grant EP/S00968X/1. All data underpinning this publication are openly available from the Univ6ersity of Strathclyde at: https://doi.org/10.15129/ 48f5ca92-c244-4426-95d8-24a22fb60e08 
The gyroklystron is a member of the gyrotron device family [10]. It is able to achieve high amplification gain at a narrow bandwidth. The gyroklystrons have been used in applications such as radar and particle accelerators. A gyroklystron operating at $48 \mathrm{GHz}$ with $2 \mathrm{MW}$ output power was designed as a potential high-power microwave source to drive a linearizer for H2020 project CompactLight X-ray free-electron laser [11, 12]. The beam-wave interaction circuit employed a three-cavity configuration. The input and intermediate cavities operated at the $\mathrm{TE}_{01}$ mode and the output cavity operates at the $\mathrm{TE}_{02}$ mode in a circular waveguide.

In this paper, a MIG was designed to generate the required electron beam to achieve a high-efficiency beam-wave interaction. The choice of MIG configuration and the solenoid system is introduced. The simulation and optimization results are presented.

\section{OPERATION OF THE MIG}

The trajectories of the electrons in a MIG are determined by both the electric and magnetic fields. The electron beam quality not only depends on the electrode structures which decide the electric field distribution, but also the coil configuration of the solenoids which determines the magnetic field. The triode-type MIG has the advantage of further tuning the beam parameter such as the beam alpha (transverse-to-axial velocity ratio, $\alpha=$ $v_{\perp} / v_{z}$ ) through the voltage at the modulation anode. For the diode-type MIG, its lack of flexibility can also be compensated by using a dedicated designed magnet system with a more complicated configuration [13, 14]. For example, additional coils at the cathode region can be used to adjust the magnetic field strength and its vector angle at the emitter surface, while keeping the field in the interaction region unchanged. In principle, the two methods do not make fundamental differences in the design stage since both are possible to achieve an optimal design if the electric and magnetic fields are wellmatched with each other.

The synthesis method has been developed to design the standard triode-type MIG [15-18]. Based on the conservation of angular momentum in the electron gun region, it has

$$
B_{z c} r_{c}^{2}=B_{0}\left(r_{g 0}^{2}-r_{L 0}^{2}\right)
$$

where $B_{z c}, B_{0}$ are the magnetic fields at the cathode and the interaction region. $r_{c}$ is the radius of the emitter and $r_{g 0}$ and $r_{L 0}$ are the guiding center and the Larmor radius at the interaction region. The equation can be written as $r_{c}^{2}=F_{m}\left(r_{g 0}^{2}-r_{L 0}^{2}\right)$ by introducing a magnetic field compression ratio $F_{m}=B_{0} / B_{z c}$.

Further derivation provides the spread in the guiding center

$$
\frac{\Delta r_{g 0}}{r_{g 0}}=\frac{\sin \phi_{c}}{\mu^{2}+1} \frac{I_{0}}{2 \pi J_{c} r_{c}^{2}}
$$

where $\phi_{c}$ is the emitter angle relative to the beam axis, $I_{0}, J_{c}$ are the beam current and the current density of the emitter. $\mu=$ $\left[\left(r_{g 0} / r_{L 0}\right)^{2}-1\right]^{-1 / 2}$ is the cylindricity parameter. At a small $\mu$ where $r_{g 0} \gg r_{L 0}$, the transverse velocity at the interaction region can be approximated as

$$
v_{\perp 0} \approx \frac{F_{m}^{3 / 2} E_{C} \cos \phi_{c}}{B_{0} \gamma_{0}}
$$

The velocity spread mainly comes from the nonuniform electric field at the emitter $\left(E_{c}\right)$ and the magnetic field $\left(B_{0} / F_{m}\right)$ due to the thickness of the emitter. The velocity spread can possibly be reduced if the electric and magnetic fields are well matched. The space charge depression voltage [19] introduces additional energy spread to the electron beam and can be another source of velocity spread. It contains depression voltage across the beam itself and the depression voltage due to the wall. The impact of depression voltage for a high energy beam is not a major issue as it can be compensated for by operating at a slightly higher beam voltage. The relation between the alpha spread and the velocity can be obtained if the space charge depression voltage of the beam is ignored. In this case, all the electrons will have the same energy in the interaction region, where $v_{\perp 0}^{2}+v_{z 0}^{2}$ is a constant value. The alpha spread is

$$
\frac{\Delta \alpha_{0}}{\alpha_{0}}=-\frac{\alpha_{0}^{2}+1}{\alpha_{0}^{2}} \frac{\Delta v_{z 0}}{v_{z 0}}=\left(\alpha_{0}^{2}+1\right) \frac{\Delta v_{\perp}}{v_{\perp 0}}
$$

The MIG is normally designed for a specific gyro-device where the beam parameters at the interaction region are determined by the interaction circuit. In this case, $\mu, I_{0}$ are known values. From eq. 2 , a small guiding center spread can be achieved by choosing large current density and emitter radius, or a small emitter angle. However, $r_{c}$ is determined by $F_{m}$ which is normally in a range of $15-25$. Its choice is usually made by experience of previous successfully operated MIGs and then validated using numerical simulation. A high $F_{m}$ will result in large Larmor radius at the cathode and leads to a larger gap distance $d_{a c}$ between the cathode and the modulation anode, otherwise, the electrons may directly strike on the modulation anode. Also the improvement by increasing $F_{m}$ is not significant after a certain value because $r_{c} \propto \sqrt{F_{m}} . J_{c}$ also has constraints imposed by the property of the material. A small emitter angle is preferred when designing the electron gun geometry.

The potential on the modulation anode can be calculated from the coaxial field theory and it is

$V_{a}=\frac{m c^{2}}{e} \frac{\ln \left(1+D_{F} \mu\right)}{\ln (1+2 \mu)}\left(\left(1+\frac{4}{\mu^{2}}\left(\frac{1+\mu}{1+2 \mu}\right)^{2} \frac{\gamma_{0}^{2}-1}{\cos ^{2} \phi_{c} r_{C}^{2} / r_{L 0}^{2}} \frac{\alpha_{0}^{2}}{\alpha_{0}^{2}+1}\right)^{0.5}-1\right)$

where $\gamma_{0}$ is the relativistic factor in the interaction region. $D_{F}=$ $d_{a c} \cos \phi_{c} /\left(\mu r_{c}\right)$ is a normalized distance factor associated with the distance between the cathode to the modulation anode. Its reasonable range is between $2.5-4.0$. A larger value will conflict with the assumption of the derivation of the synthesis method and will be difficult to achieve an optimal design, which will result in a large alpha spread.

The diode-type MIG is a special case of the standard triodetype MIG where the modulation anode is at the same voltage as the main anode. In this case in Eq. 5, a large $D_{F}$ has to be used which is not desirable for the gun design. Eq. 5 also shows that $\alpha_{0}$ increases as the increment of $V_{a}$. The electrons may be reflected back to cathode if the alpha values are too high. To avoid this, the geometry of the cathode has to change accordingly, for example, to increase $\phi_{c}$ in order to maintain a small $\alpha_{0}$. As a result, the diode-type MIG will need a significantly larger emitter angle than a triode-type one, which will result in a larger spread of the guiding center.

When a gyro-device operates with a high order mode, for example the ITER gyrotrons [20, 21], the peak electric field locates at a large radius, which also means a large guiding center. At the same $F_{m}$, a much larger emitter radius can be used as compared with a MIG designed for a lower order mode gyrotron device. The emitter thickness can therefore be minimized to reduce the alpha spread. The emitter angle is not a limitation and the diode-type MIG is a better option due to its 
simple structure. For the gyro-device operating with a low order mode and when a high beam current is required, the triode-type MIG can achieve a better beam quality and it is a better option.

\section{MIG FOR 48 GHZ GYROKLYSTRON}

A high power gyroklystron was designed as the source to drive a linearizer for the CompactLight X-ray free-electron laser. The beam-wave interaction circuit employed low order operating modes, the $\mathrm{TE}_{01}$ mode for both the input and intermediate cavities and a $\mathrm{TE}_{02}$ mode for the output cavity while minimizing mode competition. The key parameters of the gyroklystron are shown in Table I. The parameter of the electron beam generated by the MIG should satisfy the requirement of the beam-wave interaction with an alpha and guiding center spread as small as possible.

Table I key parameters of the designed gyroklystron

\begin{tabular}{|l|r|}
\hline Operating frequency $(\mathrm{GHz})$ & 48 \\
\hline Beam voltage $(\mathrm{kV})$ & 140 \\
\hline Beam current $I_{0}(\mathrm{~A})$ & 37 \\
\hline Beam alpha & 1.35 \\
\hline Magnetic field in the interaction region $B_{0}(\mathrm{~T})$ & 2.02 \\
\hline Output power $(\mathrm{MW})$ & 2.3 \\
\hline Output mode in circular waveguide & $\mathrm{TE}_{02}$ \\
\hline Gain $(\mathrm{dB})$ & 36 \\
\hline Electronic efficiency & $44 \%$ \\
\hline Beam guiding center $(\mathrm{mm})$ & 1.77 \\
\hline Pulse duration & $1.5 \mathrm{us}$ \\
\hline Maximum pulse repetition rate & $1 \mathrm{kHz}$ \\
\hline
\end{tabular}

From the analysis in the previous section, the triode-type MIG is a better choice as the gyroklystron operated with a low order mode and the beam current is large. The low order operating mode results in a small emitter radius. The small emitter angle in a triode-type MIG helps to reduce the spread in the beam. The MIG operates at high voltage. To keep the maximum electric field below the break-down threshold, the distance between the cathode and anode has to be large and leads to a large $D_{F}$ in a diode-type MIG. The triode-type MIG that has a lower voltage between the cathode and the modulation anode can achieve a suitable $D_{F}$ value to achieve a better beam quality.

The initial design of the triode-type MIG was achieved using the synthesis method. The input parameters such as the beam voltage, beam current, the guiding center at the interaction region are shown in Table I. The choice of the current density is a trade-off between the alpha spread due to the thickness of the emitter, and the emission capability of the cathode. A low current density will result in a large emitter thickness and a large alpha spread. However, a high current density will push the limit of the cathode emission capability and reduce the lifetime of the cathode. Gyroklystrons for the accelerator application requires a pulse duration of 1.5 us and the maximum pulse repetition rate of $1 \mathrm{kHz}$ (it normally operates at $400 \mathrm{~Hz}$ ). The duty circle is less than $0.2 \%$. Therefore, a relatively high current density of $20 \mathrm{~A} / \mathrm{cm}^{2}$ is feasible and it was chosen to estimate the initial parameters. Nanosized-scandia-doped dispenser cathodes with such high current density has been reported with more than 5000 hours lifetime [22]. A MIG with a current density of $30 \mathrm{~A} / \mathrm{cm}^{2}$ using a SpectraMat $612 \mathrm{X}$ scandate cathode has been designed and tested [23]. Other parameters such as the magnetic field compression ratio, and the maximum electric field were chosen as 22.5 and $7 \mathrm{kV} / \mathrm{mm}$. The parameters derived from the synthesis are shown in Table II.

Table II The initial parameters from the synthesis and the optimized parameters

\begin{tabular}{|l|l|l|}
\hline Parameters & Initial & Optimized \\
\hline Emitter radius $r_{c}$ & $7.9 \mathrm{~mm}$ & $7.9 \mathrm{~mm}$ \\
\hline Emitter current density & $20 \mathrm{~A} / \mathrm{cm}^{2}$ & $20 \mathrm{~A} / \mathrm{cm}^{2}$ \\
\hline Emitter angle $\phi_{c}$ & $30.0^{\circ}$ & $33.9^{\circ}$ \\
\hline Voltage on modulation anode $V_{a}$ & $18.7 \mathrm{kV}$ & $32.5 \mathrm{kV}$ \\
\hline $\begin{array}{l}\text { Cathode to modulation anode } \\
\text { distance } d_{a c}\end{array}$ & $15 \mathrm{~mm}$ & $15.3 \mathrm{~mm}$ \\
\hline $\begin{array}{l}\text { Magnetic field compression ratio } \\
F_{m}\end{array}$ & 25.0 & 21.93 \\
\hline Magnetic field angle $\theta$ & --- & $2.1^{\circ}$ \\
\hline
\end{tabular}

The gyroklystron operates under an ultra-high vacuum which is normally in $10^{-9}$ mbar level. However, there are still gas molecules inside, and they can be ionized during the operation. It is a complicated process and the neutralization level is not yet well understood $[24,25]$. The neutralization effect due to the ions in the high-power gyro-devices will affect the beam alpha. Therefore, it is important to have the flexibility to tune the beam parameters during the experiment. Besides the potential on the modulation anode, the magnetic field at the emitter surface provides an additional means of adjustment. The magnetic field of the gyroklystron was generated by a superconducting magnet with a closed-loop cryogenic system because the peak field $2.02 \mathrm{~T}$ requires too much drive power and cooling if normalconducting coils are used. The coil system is shown in Fig. 2.

Two sets of shim coils with the same drive current as the main coil were used to compensate for the field drop at the edge of the main coil to maintain the required flat length at the interaction region. To find an optimal magnetic field which matches with the electric field, additional magnet coils, the reverse coil 1 and 2, were used to shape to field profile at the emitter surface. Once the locations of the coils were decided, the overall magnetic field will be determined by the drive currents of the coils. Given the magnetic field strength $B_{z c}, B_{r c}$ at the emitter surface and the field at the interaction region $B_{0}$, a unique set of drive currents for the main coil and reverse coils 1 and 2 can be solved. In practice, it is more convenient to use the magnetic field angle instead of $B_{r c}$ and $F_{m}$ instead of $B_{z c}$. With first-order approximation of the off-axis magnetic field, $B_{z}(r)=B z(r=0), B_{r}(r)=-r B_{z}^{\prime} / 2$. The field angle is defined as $\theta=\operatorname{atan}\left(B_{r}(r) / B_{z}(r)\right)$.

Although the synthesis method assumes the adiabatic change of the magnetic field, and it does not consider the effect of the space charge, it provides a good starting point for parameter range scanning. The initial MIG parameters were simulated by charged-particle optics simulation software. Different tools, including MAGIC, CST Particle Studio, OPERA and TRAK [26], were simulated on an existing cusp gun, and compared with the experimental results [27, 28]. TRAK and MAGIC 2D showed close agreement in alpha spread with values comparing well with measurement. TRAK was chosen as the simulation tool due to the less computing time needed. 


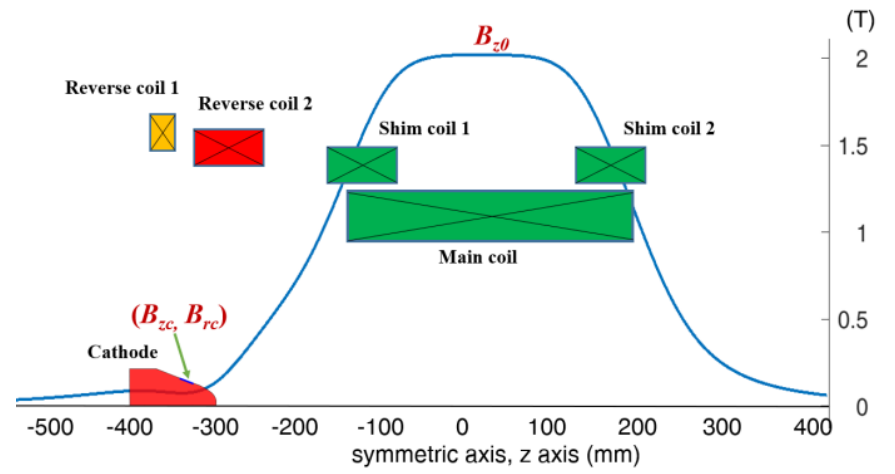

Fig. 2 the configuration of the magnet system and the magnetic field profile.

In the simulation, fine meshes of $0.05 \mathrm{~mm}$ in the radial coordinate and $0.10 \mathrm{~mm}$ in axial coordinate were used at the emitter region and meshes with $0.10 \mathrm{~mm}(\mathrm{R}) * 0.20 \mathrm{~mm}(\mathrm{Z})$ were used for the rest simulation region. The time step to advance the particle trajectories was $2.5 \times 10^{-13} \mathrm{~s}$. In the simulation, the space charge effect was calculated by iterating the electron charge deposited as the electron trajectories progressed. The simulated results showed a large alpha spread of $25 \%$. One of the reasons was the large emitter thickness due to the large emitted current needed. The nonuniform electric and magnetic at the emitter region causes the emitted electrons at different radii to experience different Lorentz forces which increases the alpha spread. The alpha spread can be reduced by optimizing the electron gun geometry and the coil configuration to find a well-matched electric and magnetic field.

Further optimizations were carried out by parameterizing the geometry, as shown in Fig. 3, and a multiple-objective genetic algorithm [29]. The same method has been used for cusp electron gun design and benchmarked with the experimental measurement [28, 30]. The geometry of the MIG was parameterized and determined by 11 parameters, together with $V_{a}$ and 3 parameters to control the magnetic field profile ( $B_{r c}, F_{m}$ and relative shift position of the coils to the cathode), there are totally 15 parameters.

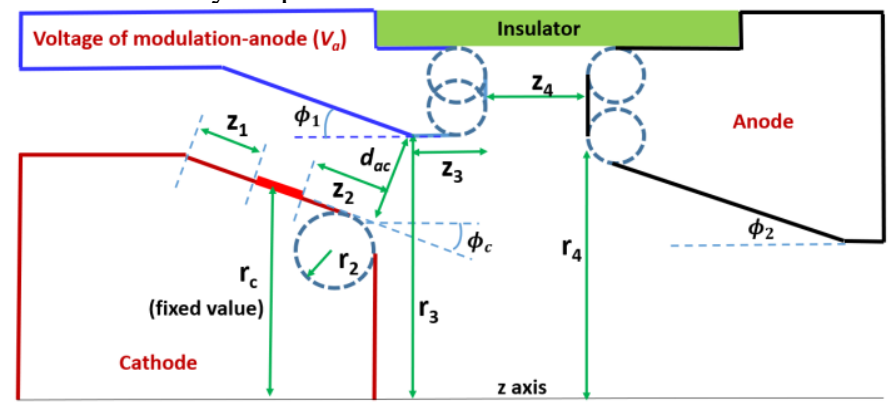

Fig. 3 The parametrized geometry of the triode-type MIG.

The particle trajectories of each parameter set were postprocessed using two evaluation functions, which was a combination of factors including the center value of the beam alpha, the guiding center, their spreads as well as the beam laminarity. The final results were chosen from the Pareto front of the values of the evaluation functions after 400 generations (each generation contains 100 populations). The final optimized values of the key parameters are also shown in Table II. The optimal magnetic field compression ratio was 21.93 , which is less than the initial value of 25 . The major reason is the large emitter thickness due to the large emitted current needed. The nonuniform electric and magnetic at the emitter region causes the emitted electrons at different radii to experience different Lorentz forces and increase the alpha spread. The effect is more significant when the magnetic field strength is smaller (equivalently large compression ratio).

\section{Simulation RESUltS}

The beam trajectories are shown in Fig. 4. A detailed plot of the trajectories of the fractional number of simulated electrons is shown in Fig. 4(b). A good laminar beam was achieved. The normalized beam current as the function of the beam alpha distribution is shown in Fig. 5. The half-width at half maximum (HWFH) alpha spread is about $8.9 \%$.

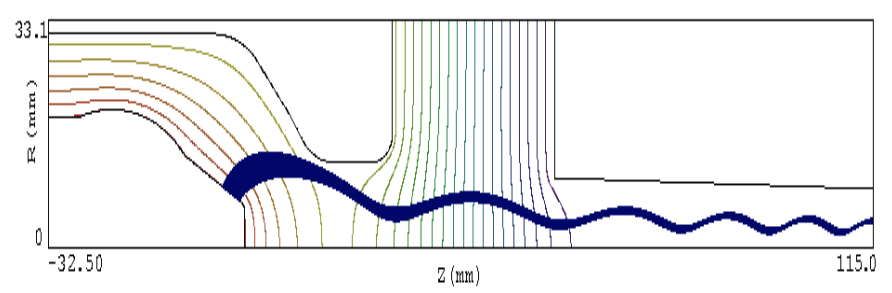

(a)

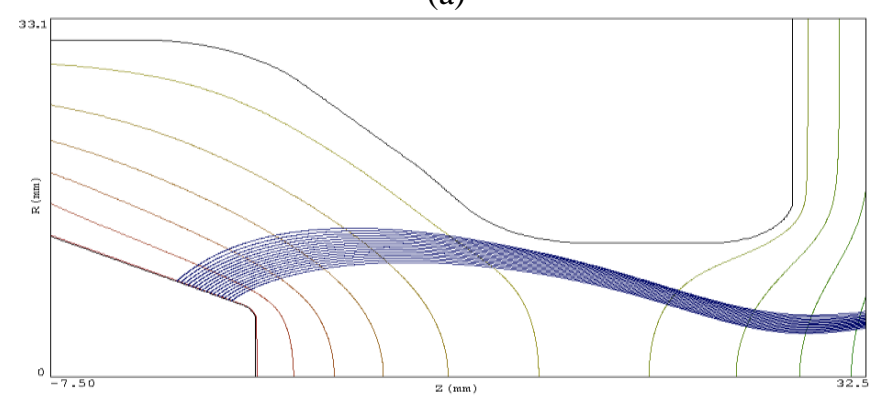

(b)

Fig. 4 (a) the electron beam trajectories in the MIG and (b), detail plot at the cathode region.

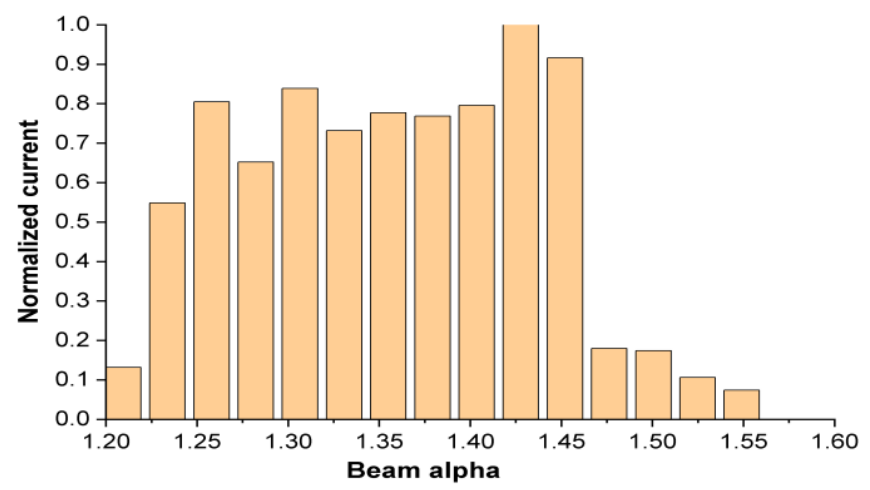

Fig. 5 the normalized beam current as of the function of beam alpha.

The beam alpha value can be adjusted by changing the applied voltage on the modulation anode and the results are shown in Fig. 6. As the voltage increases, the alpha value increases, which agrees with the analysis in section II. The 
beam alpha is relatively sensitive to the voltage at the modulation anode. With a $\sim 280 \mathrm{~V}$ variation of the voltage, the shift of the center alpha value is 0.1 . The alpha spreads have similar values at lower voltages but they increase when the voltages become larger. When the voltage is higher than $33.3 \mathrm{kV}$, although the alpha spread does not increase, the simulations show a long tail at a high alpha value range, which means a small number of electrons have high alpha values. At even higher voltages, larger than $34 \mathrm{kV}$, part of the electrons is reflected due to the alpha value being too high with the beam transportation rate no longer $100 \%$.

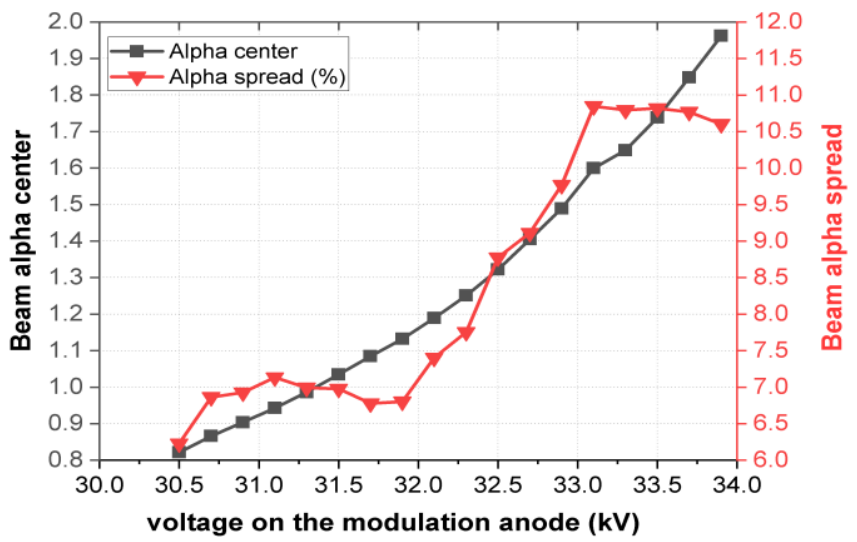

Fig. 6 the electron beam alpha as of the function of voltage on the modulation anode.

The beam alpha can also be changed by varying the compression ratio $F_{m}$, which can be implemented by shifting the position of the superconducting magnet or adjusting the drive currents of the solenoids. The simulation results are shown in Fig. 7. By shifting the position of the magnet by -2 $\mathrm{mm}$ to $2 \mathrm{~mm}$, the magnetic field compression ratio changed accordingly from 21.7 to 22.2 , the beam alpha center value changes linearly from 1.53 to 1.10 . However, there is a relatively large difference in the alpha spread when the shift distance is large. A nearly constant alpha spread at $\pm 0.25 \mathrm{~mm}$ was found from the simulation, which indicates the tolerance allowed during assembly.

Compression factor $F_{m}$

$\begin{array}{lllllllll}21.65 & 21.72 & 21.79 & 21.86 & 21.93 & 22.00 & 22.07 & 22.14 & 22.21\end{array}$

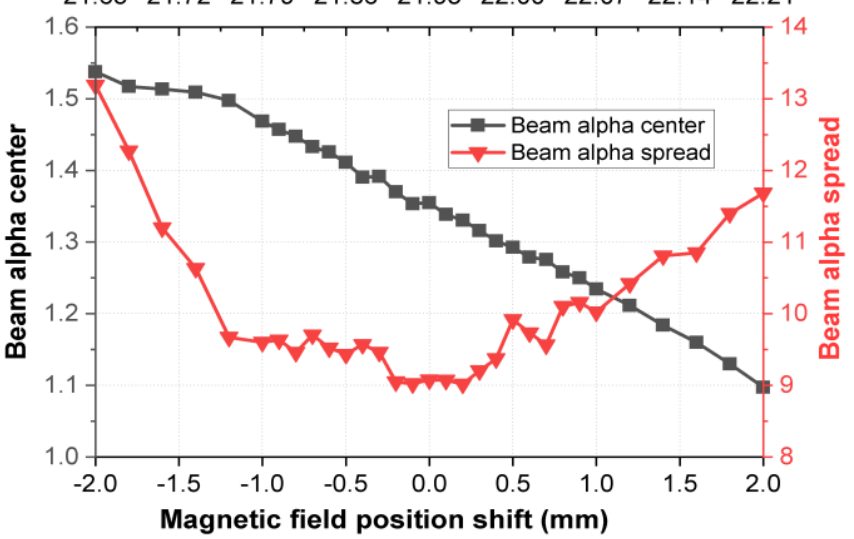

Fig. 7 the electron beam alpha as of the function of magnetic field compression factor and the shift of magnetic field profile.

Not only the $B_{z c}$ affects the beam alpha value and its spread, simulations also showed the $B_{r c}$ (which is equivalent to the field angle $\theta$ ) has a significant impact on the beam quality. From the results shown in Fig. 8, the beam alpha varies when keeping the $F_{m}$ the same and changing only the $\theta$ value. As it increases, the alpha value increases. The optimal $\theta$ is 2.1 degree, where a minimum alpha spread is achieved. The effect on the alpha spread is roughly symmetric at the optimal value. When an inadequate $\theta$ is used, the alpha spread can be huge. However, since $\theta$ is small, $B_{r c}$ is also small. It did not see a significant influence on the beam laminarity.

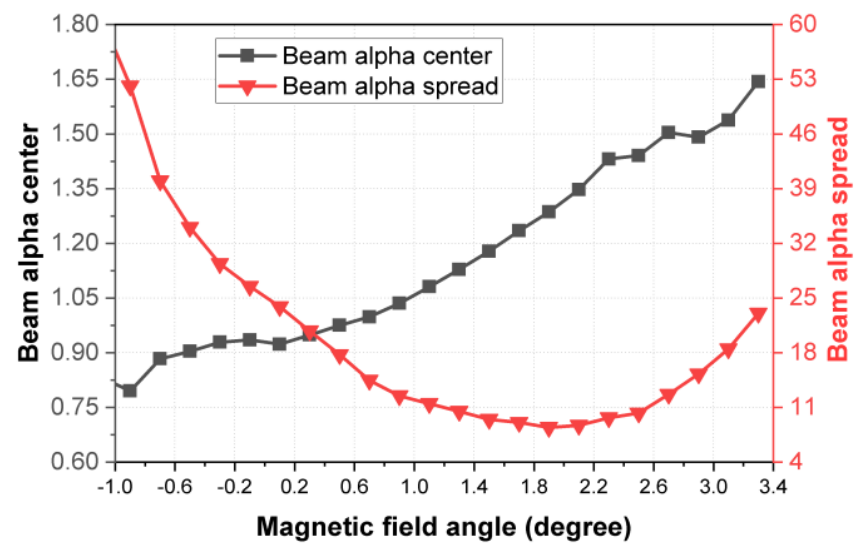

Fig. 8 the electron beam alpha as the function of the magnetic field angle at the emitter surface.

The impact of the alpha value and its spread to the beamwave interaction efficiency of the $48 \mathrm{GHz}$ gyroklystron was also investigated from the PIC simulations. The output power drops as the alpha spread increased. The trend showed a $1 \%$ increment in the alpha spread would result in a $1.55 \%$ drop in the output power. With a $9 \%$ alpha spread from the MIG gun, the output power of the gyroklystron was $1.97 \mathrm{MW}$, slightly less than the required $2 \mathrm{MW}$. Fixing at a 9\% alpha spread, the output powers of the gyroklystron at different alpha values were also shown in Fig. 9. When the beam alpha increases, the output power increases as well due to the transverse beam energy becoming larger. However, when the alpha value was larger than 1.42 , the ripple at the output power increased rapidly and the interaction became unstable.

alpha value

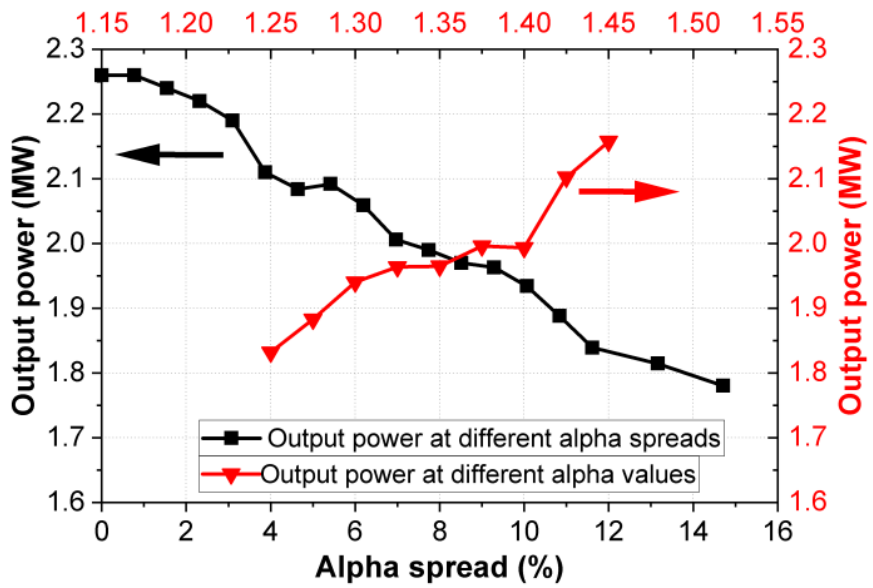

Fig. 9 the output power of the gyroklystron as the function of the alpha value and its spread. 


\section{DISCUSSION AND CONCLUSION}

In this paper, the different configurations of MIGs are analyzed and a standard triode-type MIG was designed for a 48 $\mathrm{GHz}$ gyroklystron, which is proposed to drive the linearizer for the CompactLight X-ray free electron laser (XFEL). The synthesis method was used to determine the initial parameter values and suitable ranges. The MIG was further optimized and a low alpha spread of $8.9 \%$ was achieved. The simulation results showed that the field angle at the emitter surface also played an important role in the beam quality. It changes the alpha value and has a significant effect on the alpha spread, which is beyond the theoretical prediction. Its impact on the beam laminarity is small because the magnetic field angle is small. An optimal triode type MIG requires a good match between the electric and magnetic field at the emitter area. The magnetic field at the emitter surface can be finely adjusted by introducing extra coils at the cathode region to control the magnetic field angle. The experimental study of the MIG and the $48 \mathrm{GHz}$ gyroklystron will depend upon the outcome of the XFEL project, which is currently on the research and development design stage.

\section{REFERENCES}

[1] K. R. Chu, "The electron cyclotron maser," Rev. Mod. Phys., vol. 76, no. 2, pp. 489-540, May 2004, doi: 10.1103/RevModPhys.76.489.

[2] G. S. Nusinovich, M. K. A. Thumm, and M. I. Petelin, "The Gyrotron at 50: Historical Overview," Journal of Infrared, Millimeter, and Terahertz Waves, vol. 35, no. 4, pp. 325-381, 2014/04/01 2014, doi: 10.1007/s10762-014-0050-7.

[3] J. L. Seftor, A. T. Drobot, and K. R. Chu, "An investigation of a magnetron injection gun suitable for use in cyclotron resonance masers," IEEE Trans. Electron Devices, vol. 26, no. 10, pp. 1609 1616, 1979, doi: 10.1109/T-ED.1979.19660.

[4] C. R. Donaldson et al., "A cusp electron gun for millimeter wave gyrodevices," Appl. Phys. Lett., vol. 96, no. 14, p. 141501, 2010, doi: $10.1063 / 1.3374888$.

[5] W. Lawson, "Design of low velocity - spread cusp guns for axis encircling beams," Appl. Phys. Lett., vol. 50, no. 21, pp. 1477-1479, 1987, doi: 10.1063/1.97805.

[6] I. G. Pagonakis et al., "Status of the development of the EU 170GHz/1MW/CW gyrotron," Fusion Engineering and Design, vol. 96-97, pp. 149-154, 2015/10/01/ 2015, doi: https://doi.org/10.1016/j.fusengdes.2015.02.050.

[7] S. Ruess et al., "An Inverse Magnetron Injection Gun for the KIT 2 MW Coaxial-Cavity Gyrotron," IEEE Trans. Electron Devices, vol. 63, no. 5, pp. 2104-2109, 2016, doi: 10.1109/TED.2016.2540298.

[8] K. Dong, Y. Luo, W. Jiang, H. Fu, and S. F. Wang, "Magnetron Injection Gun Design for Multifrequency Band Operations," IEEE Trans. Electron Devices, vol. 63, no. 9, pp. 3719-3724, Sep 2016, doi: 10.1109/ted.2016.2586522.

[9] V. K. Lygin, V. N. Manuilov, A. N. Kuftin, A. B. Pavelyev, and B. Piosczyk, "Inverse magnetron injection gun for a coaxial 1-5MW, $140 \mathrm{GHz}$ gyrotron," International Journal of Electronics, vol. 79, no. 2, pp. 227-235, 1995/08/01 1995, doi: 10.1080/00207219508926264.

[10] A. V. Gaponov, M. I. Petelin, and V. K. Yulpatov, "The induced radiation of excited classical oscillators and its use in highfrequency electronics," Radiophysics and Quantum Electronics, vol. 10, no. 9, pp. 794-813, 1967/09/01 1967, doi: 10.1007/BF01031607.

[11] L. J. R. Nix et al., "Demonstration of efficient beam-wave interaction for a MW-level $48 \mathrm{GHz}$ gyroklystron amplifier," Physics of Plasmas, vol. 27, no. 5, p. 053101, 2020/05/01 2020, doi: $10.1063 / 1.5144590$.

[12] L. Wang et al., "Design of a Ka-band MW-level high efficiency gyroklystron for accelerators," IET Microwaves, Antennas \&amp; Propagation, vol. 12, no. 11, pp. 1752-1757. [Online]. Available: https://digital-library.theiet.org/content/journals/10.1049/ietmap.2018.0103

[13] I. G. Pagonakis, K. A. Avramidis, G. Gantenbein, T. Rzesnicki, A. Samartsev, and J. Jelonnek, "Magnetic field profile analysis for gyrotron experimental investigation," Physics of Plasmas, vol. 24, no. 3, p. 033102, 2017/03/01 2017, doi: 10.1063/1.4977460.

[14] C. P. Yuan, T. H. Chang, N. C. Chen, and Y. S. Yeh, "Magnetron injection gun for a broadband gyrotron backward-wave oscillator," Physics of Plasmas, vol. 16, no. 7, p. 073109, 2009/07/01 2009, doi: 10.1063/1.3187903.

[15] J. Mark Baird and W. E. S. Lawson, "Magnetron injection gun (MIG) design for gyrotron applications," International Journal of Electronics, vol. 61, no. 6, pp. 953-967, 1986/12/01 1986, doi: $10.1080 / 00207218608920932$.

[16] W. Lawson, "Magnetron injection gun scaling," IEEE Trans. Plasma Sci., vol. 16, no. 2, pp. 290-295, 1988, doi: 10.1109/27.3827.

[17] W. Lawson and V. Specht, "Design comparison of single-anode and double-anode 300-MW magnetron injection gun," IEEE Trans. Electron Devices, vol. 40, no. 7, pp. 1322-1328, 1993, doi: 10.1109/16.216439.

[18] U. Singh, A. Bera, R. R. Rao, and A. K. Sinha, "Synthesized Parameters of MIG for $200 \mathrm{~kW}, 42 \mathrm{GHz}$ Gyrotron," Journal of Infrared, Millimeter, and Terahertz. Waves, vol. 31, no. 4, pp. 533541, 2010/04/01 2010, doi: 10.1007/s10762-009-9608-1.

[19] A. T. Drobot and K. Kim, "Space charge effects on the equilibrium of guided electron flow with gyromotion," International Journal of Electronics, vol. 51, no. 4, pp. 351-367, 1981/10/01 1981, doi: 10.1080/00207218108901342.

[20] J. Jelonnek et al., "From Series Production of Gyrotrons for W7-X Toward EU-1 MW Gyrotrons for ITER," IEEE Trans. Plasma Sci., vol. 42, no. 5, pp. 1135-1144, 2014, doi: 10.1109/TPS.2014.2301839.

[21] M. Thumm, "MW gyrotron development for fusion plasma applications," Plasma Physics and Controlled Fusion, vol. 45, no. 12A, pp. A143-A161, 2003/11/13 2003, doi: 10.1088/07413335/45/12a/011.

[22] F. Yang, J. Wang, Y. Wang, W. Liu, and X. Zhu, "Investigation of Nanosized-Scandia-Doped Dispenser Cathodes With Machined Surfaces," IEEE Trans. Electron Devices, vol. 63, no. 4, pp. 17281733, 2016, doi: 10.1109/TED.2016.2528580.

[23] L. R. Barnett, N. C. Luhmann, C. C. Chiu, and K. R. Chu, "Relativistic performance analysis of a high current density magnetron injection gun," Physics of Plasmas, vol. 16, no. 9, p. 093111, 2009/09/01 2009, doi: 10.1063/1.3227649.

[24] I. G. Pagonakis et al., "Electron trapping mechanisms in magnetron injection guns," Physics of Plasmas, vol. 23, no. 2, p. 023105, 2016/02/01 2016, doi: 10.1063/1.4941705.

[25] A. Schlaich et al., "Frequency-Based Investigation of Charge Neutralization Processes and Thermal Cavity Expansion in Gyrotrons," Journal of Infrared, Millimeter, and Terahertz Waves, vol. 36, no. 9, pp. 797-818, 2015/09/01 2015, doi: 10.1007/s10762015-0177-1.

[26] S. Humphries Jr, "Numerical Modeling of Space-Charge-Limited Charged-Particle Emission on a Conformal Triangular Mesh," J. Comput. Phys., vol. 125, no. 2, pp. 488-497, 1996/05/01/ 1996, doi: https://doi.org/10.1006/jcph.1996.0110.

[27] W. He, C. R. Donaldson, L. Zhang, K. Ronald, A. D. R. Phelps, and A. W. Cross, "Broadband Amplification of Low-Terahertz Signals Using Axis-Encircling Electrons in a Helically Corrugated Interaction Region," Phys. Rev. Lett., vol. 119, no. 18, p. 184801, Oct. 2017, doi: 10.1103/PhysRevLett.119.184801.

[28] L. Zhang, W. He, C. R. Donaldson, and A. W. Cross, "Investigation on the optimal magnetic field of a cusp electron gun for a W-band gyro-TWA," Physics of Plasmas, p. Submitted, 2018.

[29] W. Jiang, Y. Luo, R. Yan, and S. F. Wang, "Genetic AlgorithmBased Shape Optimization of Modulating Anode for Magnetron Injection Gun With Low Velocity Spread," IEEE Trans. Electron Devices, vol. 62, no. 8, pp. 2657-2662, Aug 2015, doi: 10.1109/ted.2015.2443068.

[30] L. Zhang, C. R. Donaldson, and W. He, "Optimization of a triodetype cusp electron gun for a W-band gyro-TWA," Physics of Plasmas, vol. 25, no. 4, p. 043120, 2018/04/01 2018, doi: $10.1063 / 1.5028262$. 